\title{
Lipid nanoparticles biocompatibility and cellular uptake in a 3D human lung model
}

\author{
Joana Magalhães ${ }^{1}$, Marina Pinheiro ${ }^{1,2}$, Barbara Drasler ${ }^{3}$, Dedy Septiadi ${ }^{3}$, Alke Petri-Fink ${ }^{3}$, \\ Susana G Santos ${ }^{4,5}$, Barbara Rothen-Rutishauser ${ }^{*, 3}$ \& Salette Reis**,1 \\ ${ }^{1}$ LAQV, REQUIMTE, Departamento de Ciências Químicas, Faculdade de Farmácia, Universidade do Porto, Portugal \\ ${ }^{2}$ Faculdade de Medicina, Universidade do Porto, Portugal \\ ${ }^{3}$ Adolphe Merkle Institute, University of Fribourg, Fribourg, Switzerland \\ ${ }^{4}$ Instituto de Investigação e Inovação em Saúde, INEB - Instituto de Engenharia Biomédica, Universidade do Porto, Porto, Portugal \\ ${ }^{5}$ Instituto de Ciências Biomédicas Abel Salazar, Universidade do Porto, Porto, Portugal \\ *Author for correspondence: barbara.rothen@unifr.ch \\ **Author for correspondence: shreis@ff.up.pt
}

\begin{abstract}
Aim: Design nanostructured lipid carriers (NLC) to facilitate drug delivery to tuberculosis-infected areas, exploiting macrophage mannose receptors and assess their uptake in a 3D human lung model. Materials \& methods: NLCs and mannosylated-NLCs were synthetized and characterized. Their uptake and biocompatibility were tested in a 3D human lung model. Results: The formulations have appropriate size (170$202 \mathrm{~nm}$ ) and morphology for lung deposition. Cell membrane integrity was maintained and no significant pro-inflammatory cytokine (IL-1 $\beta$, IL-8 and TNF- $\alpha$ ) secretion or morphological changes were observed $24 \mathrm{~h}$ post nanoparticles exposure. NLCs and mannosylated NLCs were distributed in the apical side of the lung tissue, both in macrophages and in epithelial cells. Conclusion: NLCs are biocompatible carriers and can be used for pulmonary drug delivery.
\end{abstract}

First draft submitted: 28 June 2019; Accepted for publication: 14 November 2019; Published online: 2 December 2019

Keywords: 3D lung tissue model • alternative testing strategy • nanomedicine • targeted drug delivery

Tuberculosis (TB) is a global health problem, being the leading cause of death from a single infectious agent, and one of the top ten causes of death worldwide [1]. The current TB treatment consists in a long-term multidrug combination that is associated with multiple adverse effects and low patient's compliance [2]. Therefore, improvement of therapeutic compliance is required. In the last decades, nano-based delivery systems have been explored not only to carry and protect drugs but also to efficiently deliver the drugs to the infection site, reducing the amount and frequency of dosage and thereby preventing toxicities related to therapy and improving patient's compliance [3]. These formulations represent a promising alternative for the pulmonary delivery of antibiotics, which has particular interest in TB treatment since the lungs are the primary sites of TB infection $[4,5]$.

The physicochemical properties of nanoparticles (NP), including particle size, surface and morphology are determinant factors that influence their transport and deposition within the respiratory tract [6-10]. Upon inhalation, NPs deposit mainly in the alveolar region of the lung $[11,12]$. The respiratory tract has a large epithelial surface, which is about $150 \mathrm{~m}^{2}$ [13], and a dense network of immune cells, among those macrophages and dendritic cells (DC). Lung epithelial cells play a critical role as a barrier system for inhaled particles in the respiratory system; macrophages are the main phagocytic cells, being essential for particle clearance; and DCs are the most competent antigen-presenting cells, acting as sentinels in the surveillance network of lung tissues [14,15]. The deposition of particles in the human lung is also affected by external factors such as the type of device for particle/drug delivery and the magnetic field [16].

Lipid NPs, namely nanostructured lipid carriers (NLC), represent an interesting alternative for pulmonary drug delivery, due to their biocompatibility, high drug loading capacity and stability $[17,18]$. Other advantages include the fact that NLCs size and morphology can be fine-tuned to be optimal to target a specific lung compartment [19], and their surface can be functionalized with ligands (i.e., mannose) to specifically target alveolar macrophages (AM) [20-22], key cells in TB infection [23,24]. Despite their advantages, the development of NLCs for pulmonary 
drug delivery has been hindered by concerns about their potential toxicity and biodistribution in the lungs [17,18]. Therefore, the main goal of the present study was to develop NLCs functionalized with mannose and to assess the interaction and biocompatibility in a three-dimensional (3D) co-culture model consisting of epithelial and immune cells (monocyte-derived macrophages $[\mathrm{MDM}]$ and DCs [MDDC]) mimicking the human alveolar epithelial tissue barrier as described [25].

The selection of a such a 3D human lung model is a major step further to most in vitro toxicity studies, being more realistic than the ones that are usually performed on monocultures or 2D co-cultures [7]. Moreover, advanced in vitro co-culture models provide a more cost-effective, ethical and faster alternative to in vivo models [7,26] and we already have shown that DC-specific ICAM-3-grabbing nonintegrin (DC-SIGN) conjugation to gold NPs enhanced MDDCs targeting and activation in this model [27], thus highlighting the potential of immunoengineering approaches to the targeting and activation of immune cells in the lung by nanocarriers.

In the present study, non-mannosylated NLCs and mannosylated NCLS (M-NLC) lipid NPs were developed and

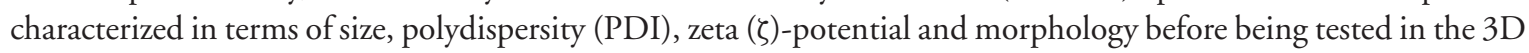
human lung model. To study their cellular uptake, the NLCs were labeled using a fluorophore (i.e., Coumarin6). The particles were applied to the lung cells either in a submerged set-up or using a pseudo air-liquid interface (ALI) approach by adding a very low volume of liquid to the lung cells to bring the exposure conditions closer to realistic situation in vivo. $24 \mathrm{~h}$ post exposure, biocompatibility and targeting efficiency of NLCs and M-NLCs was assessed using cell viability, pro-inflammatory assays and visualization of the lung tissue with confocal laser scanning microscopy.

\section{Materials \& methods}

NPs synthesis

NLCs and M-NLCs, with and without Coumarin6, were synthetized accordingly to previously published procedures (details below in Non-mannosylated lipid NPs and Mannosylated lipid NPs).

Non-mannosylated lipid NPS

NLCs were produced by ultra-sonication method with slight modifications from the method described by Vieira et al. [22]. Briefly, glyceryl palmitostearate (Precirol ${ }^{\circledR}$ ATO5, Gattefosé, Lyon, France; 66\% w/w), caprylic/capric triglyceride (Miglyol ${ }^{\circledR} 812$, Acofarma, Madrid, Spain; 13\% w/w), and polysorbate 80 (Tween ${ }^{\circledR} 80$, Merck, Darmstadt, Germany; $21 \% \mathrm{w} / \mathrm{w}$ ) were heated in a water bath up to $70^{\circ} \mathrm{C}$. When the solid lipid was fully melted, $6 \mathrm{ml}$ of preheated $\left(\mathrm{T}=70^{\circ} \mathrm{C}\right)$ Milli- $\mathrm{Q}^{\circledR}$ double-deionized water (conductivity less than $0.1 \mu \mathrm{S} \mathrm{cm}^{-1}$ ) was added to the lipid phase. This mixture was then homogenized using a probe-sonicator (Vibra-Cell model VCX 130, Sonics and Materials Inc., CT, USA) with a tip diameter of $6 \mathrm{~mm}$ at $70 \%$ amplitude for $5 \mathrm{~min}$. Nanoemulsions were left to cool down and stored at room temperature.

Coumarin6 is a lipophilic dye [28] that was used to label NLCs in order to assess their cellular uptake and internalization using fluorescence techniques. For that purpose, Coumarin6 (Sigma-Aldrich, MA, USA) was mixed with Miglyol ${ }^{\circledR} 812$ (final concentration $0.007 \% \mathrm{w} / \mathrm{w}$ ) and Coumarin6-loaded NLCs (C-NLC) were prepared using the above-mentioned approach.

\section{Mannosylated lipid NPs}

The mannose coating of NLCs (M-NLC) and C-NLCs (C-M-NLC) was performed according to Vieira et al. [22]. Briefly, $2 \% \mathrm{w} / \mathrm{w}$ of stearylamine (Sigma-Aldrich) was added to the lipid phase of the NLCs synthesis. After synthesis, a $50 \mathrm{mM} \mathrm{D-(+)-mannose} \mathrm{(Sigma-Aldrich)} \mathrm{solution} \mathrm{was} \mathrm{added} \mathrm{to} \mathrm{NLCs} \mathrm{or} \mathrm{C-NLCs,} \mathrm{in} \mathrm{50 \%} \mathrm{v/v} \mathrm{ratio.}$ The formulations were left under constant and gentle stirring for $48 \mathrm{~h}$. Further, to remove uncoated mannose and other impurities, dialysis was performed (molecular weight cut off of $12-14 \mathrm{kDa}$ ), using Milli- $\mathrm{Q}^{\circledR}$ double-deionized water (conductivity less than $0.1 \mu \mathrm{S} \mathrm{cm}^{-1}$ ), under constant and gentle stirring for $30 \mathrm{~min}$.

\section{NPs characterization}

Particle size, PDI \& $\zeta$-potential

The mean hydrodynamic particle diameter and PDI of the developed NLCs were characterized using dynamic light scattering (DLS) and $\zeta$-potentials were determined using a ZetaPALS ZetaPotential Analyzer (Brookhaven Instruments, NY, USA). Diluted NLCs ( $1: 100$ in ultrapure water) were measured at $20^{\circ} \mathrm{C}, \mathrm{pH} 5.5$, with scattering angle of $90^{\circ}$, and a dust cut-off set to 30. For mean hydrodynamic diameter and PDI, 6 runs of 2 min were 
performed at each measurement. For $\zeta$-potential determination, ten runs with ten cycles were performed at each measurement. All measurements were done in triplicates and results were expressed as mean \pm standard deviation $(\mathrm{SD})$.

\section{Transmission electron microscopy}

Transmission electron microscopy (TEM) analysis was performed to observe the morphology of the developed lipid NPs. To prepare the samples, a drop of diluted (1:100 in ultrapure water) NPs suspension was placed over a cooper-mesh grid during $2 \mathrm{~min}$, followed by negative staining with uranyl acetate for 30 seconds. Images were recorded with an accelerating voltage of $80 \mathrm{kV}$, in a JEM-1400 Transmission Electron Microscope (TEM Jeol JEM-1400; JEOL Ltd., Tokyo, Japan).

\section{Cell culture studies}

Human alveolar epithelial cell culture (A549)

The human alveolar epithelial-like cell line (i.e., human lung carcinoma cell line A549) was obtained from the American Tissue Type Culture Collection (ATCC ${ }^{\circledR}$ CCL-185 ${ }^{\text {TM }}$ ). Cells (passage number 5-20) were maintained in Roswell Park Memorial Institute (RPMI) 1640 medium (Invitrogen GmbH, Darmstadt, Germany) supplemented with 1\% L-glutamine (Invitrogen GmbH), 1\% penicillin/streptomycin (Biochrom, Berlin, Germany) and 10\% heat-inactivated fetal bovine serum (FBS; Gibco, Reinach, Switzerland). Cells were seeded at a density of $2.8 \times 10^{6}$ cells $/ \mathrm{cm}^{2}$ on polyethylene terephthalate membrane inserts for 12-well plates with high pore density and pores with $3.0 \mu \mathrm{m}$ diameter $\left(8 \times 10^{5}\right.$ pores $/ \mathrm{cm}^{2}$; surface area of $0.9 \mathrm{~cm}^{2}$; BD Biosciences, Allshwill, Switzerland). Inserts were placed in tissue culture 12-well plates and cells were grown under submerged conditions $(0.5 \mathrm{ml}$ of RPMI medium in the upper and $1.5 \mathrm{ml}$ in the lower chamber of the insert) for 5 days to achieve confluence. Media was changed every 2-3 days.

\section{Human blood MDMs \& MDDCs culture}

Human blood MDMs and MDDCs were isolated from buffy coats provided by the Transfusion Blood Bank (Blutspendedienst SRK Bern AG, Bern, Switzerland), according to the method described by Sallusto and coworkers [29], with the adaptation of using CD14 magnetic beads (Milteny Biotech, Bergisch Gladbach, Germany) for monocytes isolation [30]. Isolated blood monocytes were cultured for 7 days at a density of $10^{6}$ cells $/ \mathrm{ml}$ in RPMI 1640 medium supplemented with $1 \%$ L-glutamine, $1 \%$ penicillin/streptomycin and $10 \%$ heat-inactivated FBS. For MDMs differentiation, $10 \mathrm{ng} / \mathrm{ml}$ of granulocyte macrophage colony-stimulating factor (GM-CSF; Sigma Aldrich ) were added to the culture medium. MDDCs differentiation was performed in presence of $10 \mathrm{ng} / \mathrm{ml}$ of IL-4 (Sigma Aldrich $\mathrm{GmbH}$ ) and $10 \mathrm{ng} / \mathrm{ml}$ of GM-CSF (Sigma Aldrich GmbH) for 6-7 days [30].

\section{Triple cell co-culture model}

The triple cell co-culture model was performed based on the protocol described by Rothen-Rutishauser et al. [25]. Briefly, the inserts containing A549 cells were placed in a petri dish upside down, and the cells at the bottom of the membrane were gently removed with a cell scraper. MDDCs $\left(5.95 \times 10^{4} \mathrm{cells} / \mathrm{cm}^{2}\right)$ were then pipetted onto the bottom side of the inserts and incubated for $1 \mathrm{~h}$ at $37^{\circ} \mathrm{C}$ and $5 \% \mathrm{CO}_{2}$. Afterward, the inserts were placed back into the 12-well plates containing $1.5 \mathrm{ml}$ of pre-heated supplemented RPMI medium at the bottom. Finally, MDMs $\left(1.19 \times 10^{4}\right.$ cells $\left./ \mathrm{cm}^{2}\right)$ were gently added on the top of the A549 cells. The cells were then incubated for $24 \mathrm{~h}$ at $37^{\circ} \mathrm{C}$ and $5 \% \mathrm{CO}_{2}$ until NLCs-exposures (if submerged) or placement of samples at the air-liquid interface (for pseudo-ALI).

\section{NPs exposure}

The cells were exposed to mannosylated and non-mannosylated lipid NPs $\left(278 \mu \mathrm{g} / \mathrm{cm}^{2}\right)$, with and without the fluorophore, under submerged and pseudo-ALI conditions. For submerged exposures, after $24 \mathrm{~h}$ post incubation, the media in the lower chamber of the insert was replaced with $1.5 \mathrm{ml}$ of fresh supplemented RPMI, and $0.5 \mathrm{ml}$ NPs suspension $(0.5 \mathrm{mg} / \mathrm{ml})$ was gently added on the top of MDMs and A549 cells. In pseudo-ALI exposures, cells were pre-exposed to ALI by removing the medium in the upper compartment and replacing the medium from the lower chambers with $0.6 \mathrm{ml}$ of fresh supplemented RPMI, for $24 \mathrm{~h}$. Then, a thin layer of particle suspension $(0.05 \mathrm{ml} ; 5 \mathrm{mg} / \mathrm{ml})$ was gently added apically to the cells cultivated at ALI. Both submerged and pseudo-ALI exposures were performed by incubating the cells with NPs for $24 \mathrm{~h}$ at $37^{\circ} \mathrm{C}$ and $5 \% \mathrm{CO}_{2}$. 


\section{Cytotoxicity assessment}

The LDH release was assessed as indicator for cytotoxicity based on the release of LDH into the medium due to plasma membrane permeabilization as a result of cell death [31]. For this purpose, the medium in the lower chamber of the inserts was collected after exposures to NPs and analyzed using an LDH cytotoxicity detection kit (Roche Applied Science, Germany) according to the manufacturer's instructions. To determine the LDH activity, absorbance was read at $490 \mathrm{~nm}$ (reference wavelength at $630 \mathrm{~nm}$ ) using a microplate reader (Bio-Rad, Cressier, Switzerland). Samples were measured in triplicates and each sample absorbance was corrected by subtracting medium absorbance. Co-cultures exposed to $0.2 \%$ Triton X-100 (Sigma Aldrich GmbH) in phosphate buffer saline (PBS), for $24 \mathrm{~h}$ were used as positive control. $\mathrm{LDH}$ values were expressed relative to negative control, in other words, untreated cells. For untreated cells, the same volume of supplemented RPMI medium was added to the upper chamber of the insert but without NPs.

\section{Pro-inflammatory response}

The pro-inflammatory response of the cells after NPs exposure was assessed by quantifying the amount of proinflammatory mediators, IL-1 $\beta$, IL- 8 and TNF- $\alpha$ in the supplemented RPMI, using the respective DuoSet ELISA Development Kit (R\&D Systems, Zug, Switzerland) according to the manufacturer's instructions. For IL-8 quantification, samples were diluted (1:10 in reagent diluent) as the cytokine is already released at a basal level in untreated cultures. Absorbance was read at $450 \mathrm{~nm}$ using a microplate reader (Bio-Rad, CA, USA). Samples were measured in triplicates, and each sample absorbance was corrected by subtracting medium absorbance. Untreated cells were used as negative control, and cells treated with lipopolysaccharide (LPS, from Escherichia coli 055:B5 strain, $1 \mu \mathrm{g} / \mathrm{ml}$ in supplemented RPMI; Sigma Aldrich were used as a positive pro-inflammatory assay control.

\section{Cell labeling}

In the first staining procedure, the cultures were fixed with $4 \%$ paraformaldehyde (PFA; Sigma Aldrich GmbH) in PBS for $15 \mathrm{~min}$, and then treated with $0.1 \mathrm{M}$ glycine (Sigma Aldrich GmbH) in PBS for 5 min. Before staining, cells were permeabilized with $0.2 \%$ Triton X-100 (Sigma Aldrich GmbH) in PBS for 15 min. MDMs were stained for 90 min with the primary antibody mouse anti-human 25F9 at a 1:100 dilution in PBS (Clone eBio25F9; Thermo Fisher Scientific, Bremen, Germany). The secondary staining was performed using goat anti-mouse Alexa 647 (Polyclonal; Abcam, Cambridge, UK) as a secondary detection antibody at a 1:50 dilution in PBS; rhodaminephalloidin (Thermo Fisher Scientific) at a 1:50 dilution to stain the F-actin cytoskeleton; and DAPI ([1 mg/ml in 0.3\% Triton X-100 in PBS; Sigma Aldrich, MA, USA) to stain the nucleus, for $2 \mathrm{~h}$ in the dark. After staining, cells were washed three-times with PBS and then, the membranes were cut with a scalpel into two pieces. For optical analysis, samples were embedded in glycergel (DAKO Schweiz AG, Baar, Switzerland). One piece of each insert membrane was turned upside down to investigate the cells grown on the basal side of membrane inserts.

In the second approach, the three different cell types in the co-culture model were pre-stained before exposure to NPs using Hoechst 33342 (Invitrogen GmbH, Darmstadt, Germany) and the Vybrant ${ }^{\text {TM }}$ multicolor cell labeling kit (Thermo Fisher Scientific) prior to the co-culture composition, following the protocol described by Septiadi et al. [32]. Briefly, MDDCs and MDMs were stained with Vybrant ${ }^{\circledR}$ DiI and Vybrant ${ }^{\circledR}$ DiD, respectively $(5 \mu \mathrm{l} / \mathrm{ml}$ of cell suspension), and incubated for $30 \mathrm{~min}$ at $37^{\circ} \mathrm{C}$ and $5 \% \mathrm{CO}_{2}$. Cells were centrifuged and washed three times with RPMI 1640, prior to seeding. In the meantime, the nuclei of A549 cells on the insert were stained using Hoechst $33342\left(10 \mu \mathrm{l}\right.$ in $1 \mathrm{ml}$ of RPMI 1640) and incubated for $30 \mathrm{~min}$ at $37^{\circ} \mathrm{C}$ and $5 \% \mathrm{CO}_{2}$. Cells were washed three-times with RPMI 1640, and finally the co-cultures were composed and exposed to NLCs as previously described. After $24 \mathrm{~h}$ of NPs exposure, cells were washed three-times, fixed using 4\% PFA and prepared for optical analysis, as previously explained.

\section{Fluorescence imaging: laser scanning microscopy}

The samples were visualized using Zeiss LSM 710 confocal laser scanning inverted microscope (Axiovert $200 \mathrm{M}$, Lasers: 405, 488 and $633 \mathrm{~nm}$ ) with a $63 \times$ objective lens (oil immersion, NA = 1.3; Zeiss GmbH, Munich, Germany). Different fluorophores (i.e., Hoechst 33342/DAPI, Coumarin6, Vybrant ${ }^{\circledR}$ DiI/rhodamine-phalloidin, and Vybrant ${ }^{\circledR}$ DiD/Alexa647) were excited sequentially at 405, 458, 561 and $633 \mathrm{~nm}$, and their emissions were collected correspondingly by the detector with the frame size 512 pixel $\times 512$ pixel $(134.95 \mu \mathrm{m} \times 134.95 \mu \mathrm{m})$. Images were acquired in the plane scan mode or in a z-stack mode with the slice thickness of $0.5 \mu \mathrm{m}$. Image pro- 


\begin{tabular}{|c|c|c|c|}
\hline $\begin{array}{l}\text { Characterization of the developed lipid } \\
\text { nanoparticles }\end{array}$ & Size $(\mathrm{nm})$ & PDI & $\zeta$-potential $(\mathrm{mV})$ \\
\hline C-NLC & $170 \pm 6$ & $0.18 \pm 0.01$ & $-26 \pm 2$ \\
\hline M-NLC & $202 \pm 7$ & $0.17 \pm 0.02$ & $38 \pm 2$ \\
\hline
\end{tabular}

Data are expressed as mean \pm SD $(n=3)$.

C-NLC: Coumarin6-loaded nanostructured lipid carrier; C-M NLC: Coumarin6-loaded mannosylated nanostructured lipid carrier; M-NLC: Mannosylated nanostructured lipid carrier; NLC: Nanostructured lipid carrier; PDI: Polydispersity index.

cessing and visualization were performed using the $3 \mathrm{D}$ multichannel image processing software IMARIS (Bitplane AG, Zurich, Switzerland).

\section{Quantification of lipid NPs uptake using image processing}

15 different images in the apical side of the samples (C-NLCs and C-M-NLCs containing samples) were acquired using z-stack acquisition with slice thickness of $1 \mu \mathrm{m}$. These $\mathrm{z}$-stack images were then processed as mean intensity projection using Zen software (Zeiss GmbH, Munich, Germany). Particle tracking and counting were done using TrackMate plugin in Fiji (NIH, USA) [33]. Briefly, Laplacian of Gaussian filter with a sigma value suited to the size of particle agglomerate of $1 \mu \mathrm{m}$ (i.e., this estimated size is limited to the resolution of fluorescence confocal microscope) and intensity threshold of 25 was used as two main parameters to count the number of particles in the images. Data (i.e., number of agglomerates per $1000 \mu \mathrm{m}^{2}$ ) are shown as box chart.

\section{Statistical analysis}

Statistical comparisons of the mean of the different groups were performed using the ordinary one-way ANOVA followed by Dunnet's multiple comparison test relative to negative control cells. To compare the number of agglomerates, one-way ANOVA followed by Tukey's test was performed. A p-value $<0.05$ was considered statistically significant. The analyses were performed using the GraphPad Prism6 software program (GraphPad Software Inc., CA, USA) and Origin (OriginLab Corporation, MA, USA). Data were expressed as mean \pm SD.

\section{Results}

\section{Synthesis \& characterization of lipid NPs}

The NLCs composition and synthesis method were chosen according to preliminary formulation studies performed by the authors [22,34-36]. After synthesis, the developed NLCs were characterized in terms of size, PDI and $\zeta$-potential (Table 1). The mean hydrodynamic particle size of non-mannosylated NLCs and C-NLCs were $182 \pm 8 \mathrm{~nm}$ and $170 \pm 6 \mathrm{~nm}$, respectively. Regarding the mannosylated NLCs, the values were $202 \pm 7 \mathrm{~nm}$ for M-NLCs, and $192 \pm 6 \mathrm{~nm}$ for C-M-NLCs. As expected, the mannosylation process led to an increase in NPs diameter (pvalue $>0.05$ ). Regarding the labeling approach, there were no statistically significant differences in hydrodynamic particle size between nonlabeled and Coumarin6 labeled NLCs ( $\mathrm{p}$-value $>0.05$ ), which confirms that the dye functionalization did not alter the overall NLCs hydrodynamic size. PDI values were below 0.2 and all the formulations had high absolute $\zeta$-potential values (Table 1). Moreover, storage stability studies were performed and the developed NLCs were stable during at least 3 months at $20^{\circ} \mathrm{C}$ (Supplementary Figure 1).

To observe the morphology of the developed NPs, TEM analysis was performed. Results reveal spherical particles, uniform in shape, with no visible aggregation for both NLCs (Figure 1A) and M-NLCs (Figure 1B). The mean diameter of NPs was in the range of $200 \mathrm{~nm}$.

Biocompatibility \& pro-inflammatory response in the 3D human lung model upon exposure to NPs 3D-rendered images of the 3D human lung model were reconstructed from acquired Z-series of confocal images. Epithelial cells formed a monolayer (Figure 2A; blue); MDMs were localized on the top of the epithelial monolayer (Figure 2A; red), and MDDCs at the basal surface of the insert (Figure 2A; yellow). The cells were exposed to nonmannosylated (NLCs and C-NLCs) and mannosylated (M-NLCs and C-M-NLCs) lipid NPs under submerged and pseudo-ALI conditions $\left(278 \mu \mathrm{g} / \mathrm{cm}^{2}\right)$ for $24 \mathrm{~h}$. The cytotoxic effect of NPs exposure was assessed through 

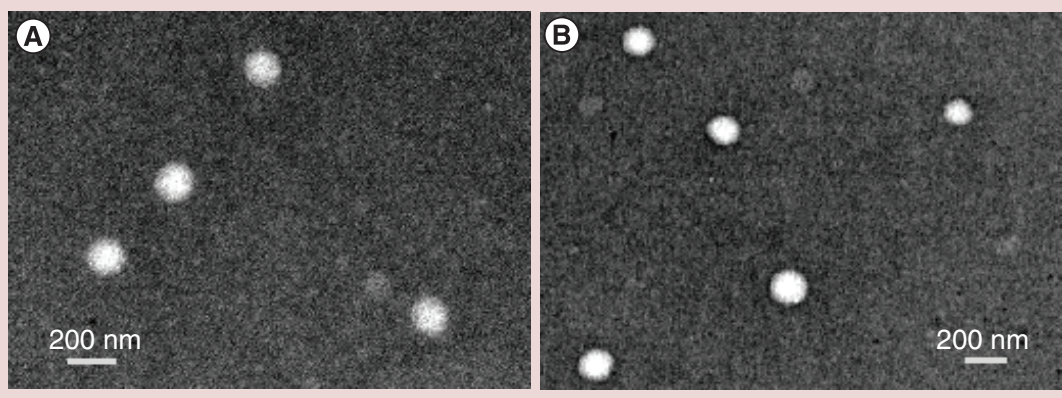

Figure 1. Morphology of the developed lipid nanoparticles. Transmission electron microscopy images of (A) nanostructured lipid carriers and (B) mannosylated-nanostructured lipid carriers, at 50,000× magnification. The white bar represents $200 \mathrm{~nm}$.

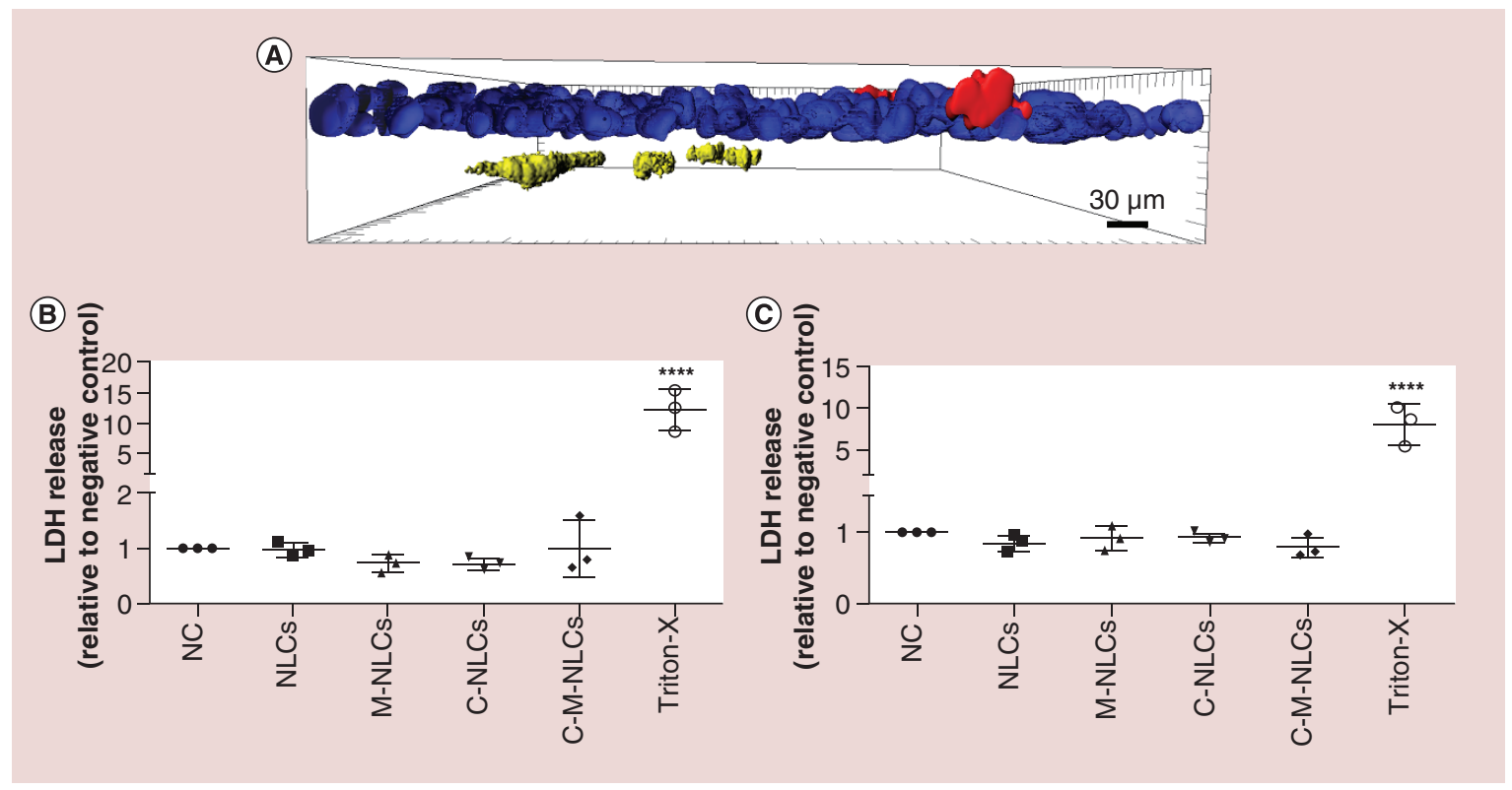

Figure 2. Cell layer integrity and LDH release following lipid nanoparticles exposures to the 3D human lung model. (A) 3D-rendered fluorescence confocal micrograph of the cell culture model. Macrophages were stained using Vybrant $^{\circledR}$ DiD (red), nuclei of epithelial cells were stained using Hoechst 33342 (blue) while monocyte-derived dendritic cells were labeled using Vybrant ${ }^{\circledR}$ Dil (yellow). The cell cultures were exposed to different nanostructured lipid carriers by (B) submerged and (C) pseudo air-liquid interface exposures. Cell membrane rupture was evaluated by quantification of LDH release in cell culture medium after $24 \mathrm{~h}$ post nanoparticles exposure. Data of three donors are represented in scatter plots, where the horizontal line indicates the mean. The values are expressed as a fold change relative to untreated cells of the respective donor. Statistical analysis was performed using the one-way ANOVA followed by Dunnett's multiple comparison test relative to NC. $* * * * p<0.0001$

C-NLC: Coumarin6-loaded nanostructured lipid carrier; C-M NLC: Coumarin6-loaded mannosylated nanostructured lipid carrier; LPS: Lipopolysaccharide; M-NLC: Mannosylated nanostructured lipid carrier; NLC: Nanostructured lipid; NC: Negative control.

the quantification of LDH release to the cell culture medium, which reflects cell membrane rupture. Results revealed that NLCs did not alter the cell membrane integrity of the cells, neither under submerged nor pseudo-ALI conditions (Figure $2 \mathrm{~B} \& \mathrm{C}$ ). Cells exposed to $0.2 \%$ Triton X-100 for $24 \mathrm{~h}$ were used as a positive control for membrane rupture (significant LDH release observed; Figure 2B \& C).

To evaluate the pro-inflammatory response of the cells after NLCs exposure, the release of different cytokines was measured. LPS-exposed cells were used as a positive control for induction of pro-inflammatory response. Exposure 
(A)
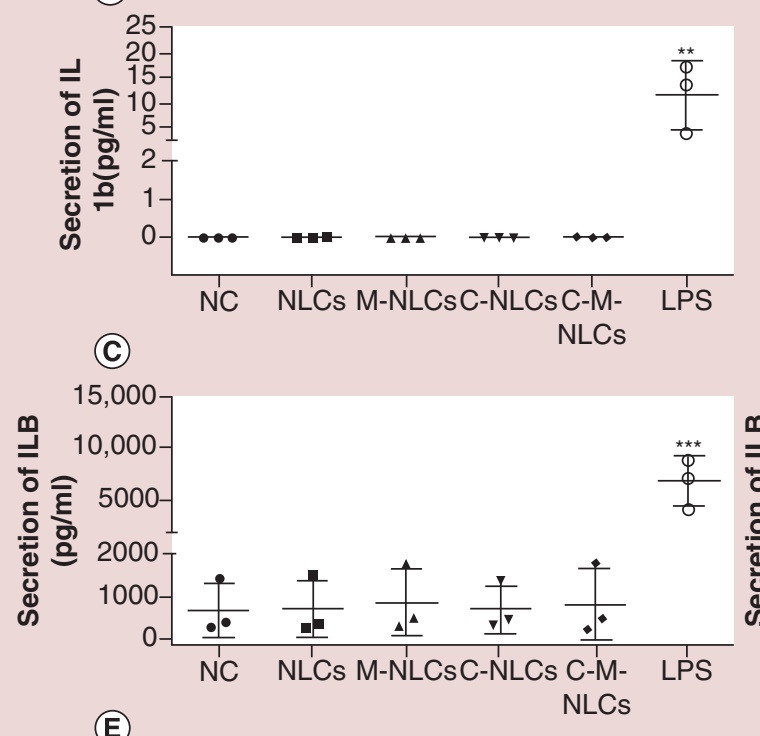

(E)

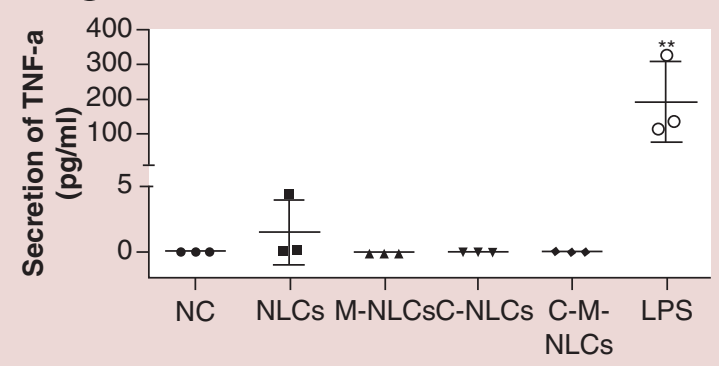

(B)
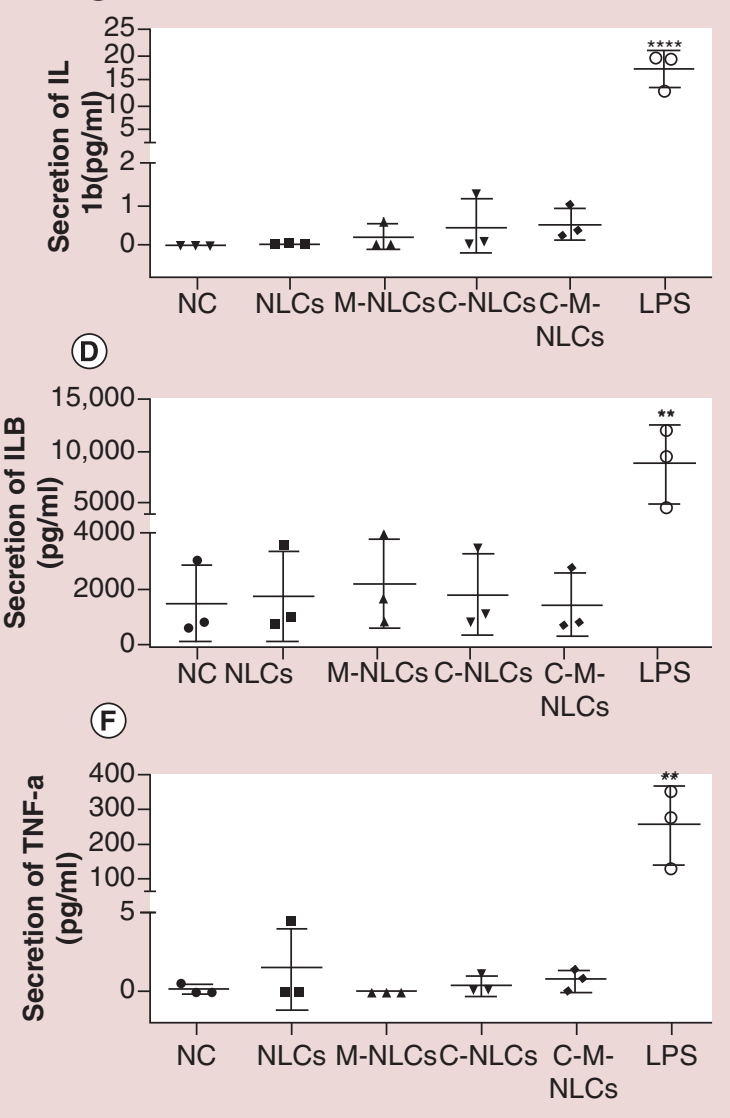

Figure 3. Pro-inflammatory response of the 3D lung model upon lipid nanoparticles exposures. Cells were exposed to different nanostructured lipid carriers by (A, C \& E) submerged and (B, D \& F) pseudo air-liquid interface exposures. Secretion of the pro-inflammatory chemokines (A \& B) IL-1 $\beta$, (C \& D) IL-8 and (E \& F) TNF- $\alpha$ to the cell culture medium after $24 \mathrm{~h}$ post nanoparticles exposure (shown relative to untreated cells of the respective donor). Untreated cells were used as negative control (NC) and LPS-exposed as positive control. For IL-1 $\beta$ and TNF- $\alpha$ all the samples with the exception of LPS-exposed cells were below the detection limit of the instrument. Data of three donors are represented in scatter plots, where the horizontal line indicates the mean. In all cases, comparisons were performed using the ordinary one-way ANOVA followed by Dunnett's multiple comparison test relative to untreated cells (NC). $* * p<0.01 ; * * * p<0.001 ; * * * * p<0.0001$

C-NLC: Coumarin6-loaded nanostructured lipid carrier; C-M NLC: Coumarin6-loaded mannosylated nanostructured lipid carrier; LPS: Lipopolysaccharide; M-NLC: Mannosylated nanostructured lipid carrier; NLC: Nanostructured lipid; NC: Negative control.

to all tested NLCs for $24 \mathrm{~h}$ did not induce statistically significant increase of the investigated cytokines, IL-1 $\beta$, TNF- $\alpha$ and IL-8, released in the cell culture media, compared with untreated cells (NC; Figure 3). An increased amount of all the tested cytokines was observed for the LPS-stimulated samples, which supports the responsiveness of the model to pro-inflammatory stimulus. IL- $1 \beta$ and TNF- $\alpha$ were below the detection limits of the experimental set-up for untreated cells and all the exposure samples (Figure 3A \& B, E \& F), respectively. The levels of IL-8 secretion (Figure $3 \mathrm{C} \& \mathrm{D}$ ) were higher than the ones obtained for IL-1 $\beta$ (Figure $3 \mathrm{~A} \& \mathrm{~B}$ ), and TNF- $\alpha$ (Figure $3 \mathrm{E}$ $\& \mathrm{~F})$.

Lung cell morphology \& cellular uptake of lipid NPs

Cellular uptake of fluorescently labeled NLCs was first evaluated under submerged conditions and then the approach was moved towards a more realistic exposure scenario, in other words, at the pseudo-ALI conditions. In both the approaches, the co-culture model was exposed to fluorescently labeled NLCs, either with or without mannose, 


\section{(A)}

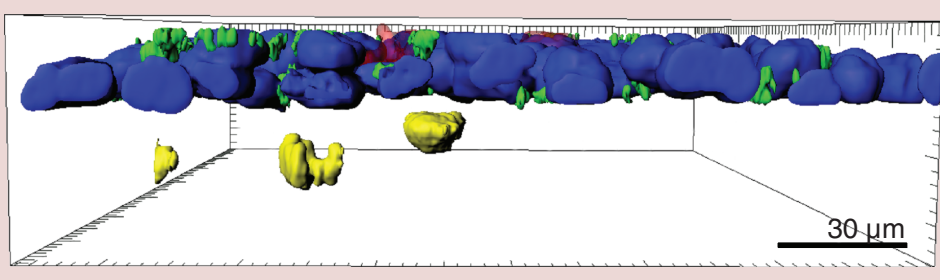

(B)

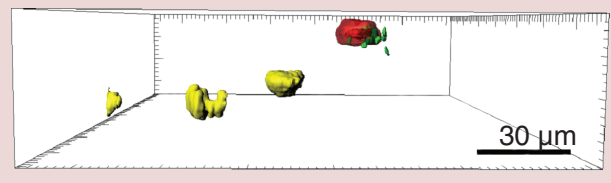

(C)

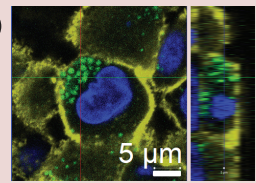

Figure 4. Fate of lipid nanoparticles in the 3D human lung cell model. The cells were exposed to both Coumarin6-loaded nanostructured lipid carriers and Coumarin6-loaded mannosylated nanostructured lipid carriers. (A) 3D-rendered fluorescence confocal micrograph shows that $24 \mathrm{~h}$ post exposure Coumarin6-loaded mannosylated nanostructured lipid carriers (green; Coumarin6) are mostly associated to monocyte-derived macrophages (red) and epithelial cells (blue; Hoechst 33342), but not in the monocyte-derived dendritic cells. Z-projection images reveal the intracellular distribution of Coumarin6-loaded mannosylated nanostructured lipid carriers in (B) monocyte-derived macrophages (red) and (C) epithelial cells. Monocyte-derived macrophages were stained using Vybrant ${ }^{\circledR}$ DiD (red) while cytoskeletal architectures of epithelial cells were stained using rhodamine-phalloidin (yellow).

for $24 \mathrm{~h}$. An example of NLC-model association is presented upon exposure to fluorescently labeled M-NLCs at submerged conditions (Figure 4A). A total of $24 \mathrm{~h}$ post exposure, the particles (in green) were mostly distributed in the apical side of the lung tissue, both in the MDMs (in yellow) and in epithelial cells (in blue). There were no NLCs observed in the basal side of the model indicating absence of NPs translocation across the alveolar epithelial barrier tissue, under the investigated exposure conditions. Our results also further confirm the intracellular distribution of NLCs inside MDMs and epithelial cells (Figure 4B \& C).

To check if cell cultures exposed to NLCs under submerged or pseudo-ALI will maintain the cell (monolayer barrier) morphology, the cytoskeletal parts of the fixed co-culture model (F-actins) were stained. Post $24 \mathrm{~h}$ of NPs incubation, we did not observe any morphological changes of the monolayer exposed to C-NLCs (Figure 5A \& C) or to C-M-NLCs (Figure 5B \& D).

Particle tracking and counting based on image processing was used to test whether the co-culture model possess different response to non-mannosylated and mannosylated NLCs. Briefly, 15 z-stack images were acquired for each sample from randomly chosen area. These images were processed using mean intensity projection and the corresponding micrographs were subjected to particle counting. Due to the resolution of images, single particle counting is not accessible. In this case, only agglomerates in size of $1 \mu \mathrm{m}$ were taken into account during counting. The representative mean intensity projection images are shown in Figure 6A-C. Quantitative analysis of numbers of agglomerates per $1000 \mu \mathrm{m}^{2}$ are depicted in Figure 6C. Our statistical analysis however, shows no significant differences in term of particle number (i.e., agglomerates) of the two tested nanoformulations.

\section{Discussion}

The main aim of the present work was to design NLCs to facilitate drug delivery to TB-infected areas. For that purpose, mannose coating of NLCs was performed as an active targeting approach to take advantage of the mannose receptors expressed by AMs, thus increasing the nanocarriers selectivity to Mycobacterium tuberculosis infected areas and cells [21,22]. After synthesis, non-mannosylated and mannosylated NLCs were characterized regarding their size, PDI, $\zeta$-potential and morphology. The increase in NLCs diameter observed after mannosylation was in agreement with the results obtained in previous studies [20,37]. NLCs and M-NLCs were labeled with Coumarin6 to assess their cellular uptake using fluorescence techniques, and this did not alter their hydrodynamic size. Overall, the mean particle size of the herein developed nanoformulations is optimal for deposition in the alveolar region since they are not larger enough (higher than $5 \mu \mathrm{m}$ ) to be preferentially deposited in the upper airways, or small enough (smaller than $0.05 \mu \mathrm{m}$ ) to be accumulated in the nasopharyngeal area [19,38]. Moreover, particles smaller than $1 \mu \mathrm{m}$ 

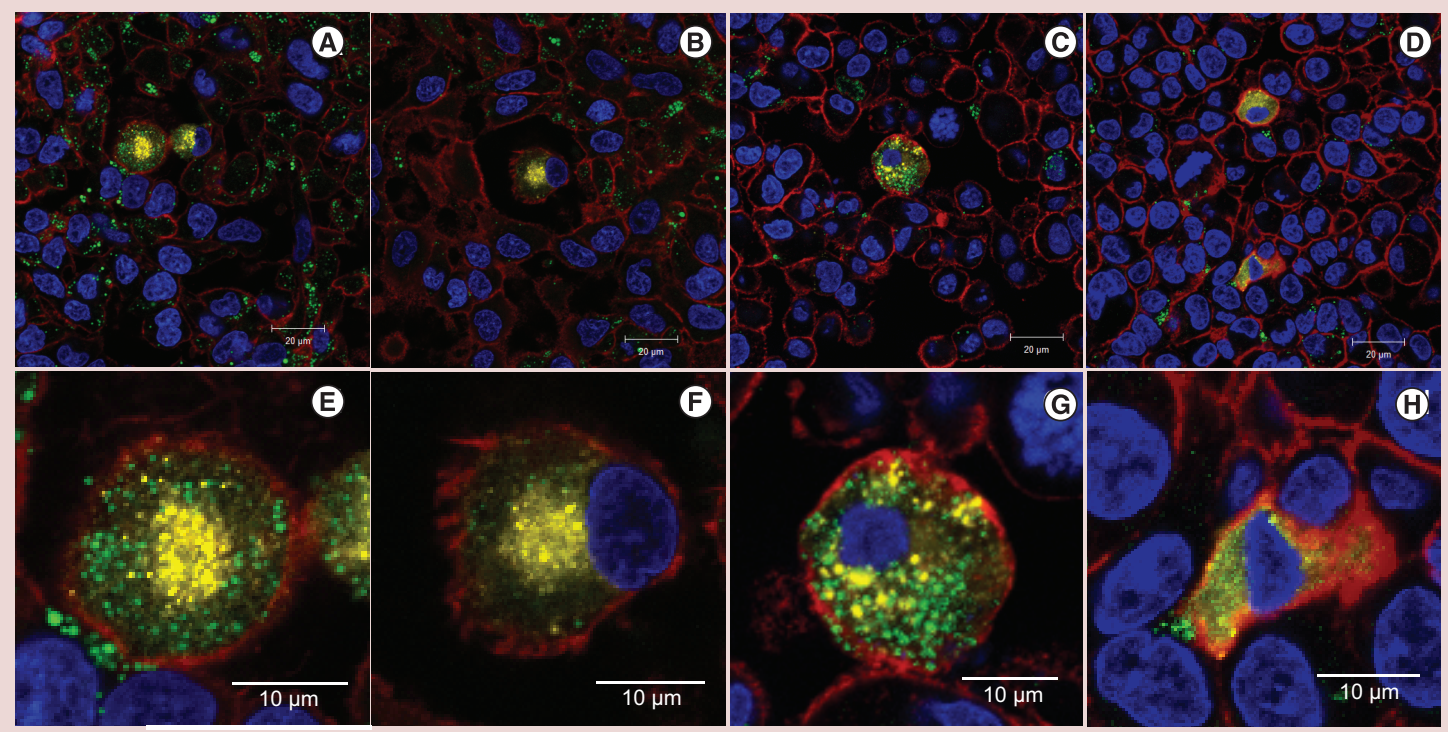

Figure 5. Nanoparticles-cells interaction in the 3D human lung model. Confocal laser scanning micrographs of the apical sides of the cell cultures exposed to (A \& C) Coumarin6-loaded nanostructured lipid carriers and (B \& D) Coumarin6-loaded mannosylated nanostructured lipid carriers using (A \& B) submerged exposures and (C \& D) pseudo air-liquid interface conditions. (E-H) Zoom of monocyte-derived macrophages present in A-H images. Fluorescence labeling: nuclei in blue (DAPI), nanoparticles in green (Coumarin6), cytoskeleton (F-actin) in red (rhodamine-phalloidin) and monocyte-derived macrophages in yellow (Alexa 647).
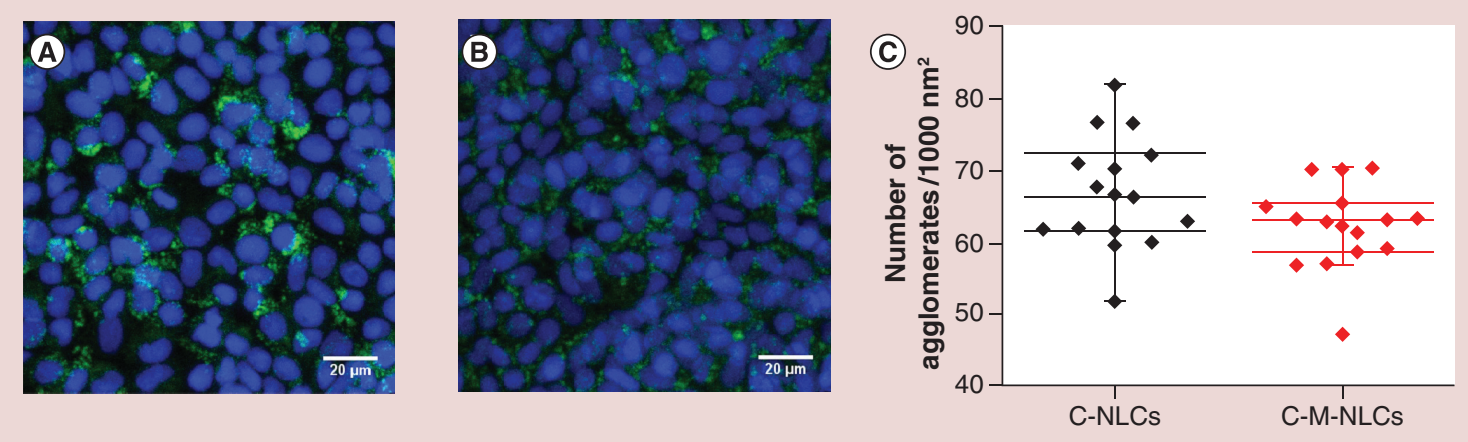

Figure 6. Lipid nanoparticles cellular uptake in the 3D human lung model. Mean intensity projection images showing distribution of (A) Coumarin6-loaded nanostructured lipid carriers (green) and (B) Coumarin6-loaded mannosylated nanosctructured lipid carriers (green) on the cells. (C) Quantitative analysis of lipid nanoparticles association in the cells. Data are shown as number of agglomerates (diameter $\sim 1 \mu \mathrm{m}$ ) per $1000 \mu \mathrm{m}^{2}$. One way-ANOVA analysis (Tukey's test, $\mathrm{p}<0.05$ ) was performed, however the results shows no statistically significant different between the two tested nanoparticles.

C-NLC: Coumarin6-loaded nanostructured lipid carrier; C-M NLC: Coumarin6-loaded mannosylated nanostructured lipid carrier.

tend to reach the tertiary bronchi and bronchioles, being the NPs between 50 and $200 \mathrm{~nm}$ desired for maximized drug localization upon administration by inhalation $[39,40]$.

PDI values were below 0.2 for all formulations, suggesting a uniform distribution of NLCs [40]. Regarding their surface charge, NLCs and C-NLCs have a highly negative $\zeta$-potential, while M-NLCs and C-M-NLCs possessed a positive $\zeta$-potential. This result was in agreement with our previous study [22] and confirms that the addition of stearylamine residues in the mannosylation process of NLCs confers a positive charge to the functionalized 
NLCs [20]. All the formulations have high absolute $\zeta$-potential values, indicating that they are physically stable after synthesis [41].

Confocal laser scanning microscope (LSM) combined with digital image restoration (i.e., 3D rendering) was employed for visualization of the epithelial tissue morphology, localization of the various cells and barrier structure. For this purpose, individual cell types were pre-labeled with cell markers before construction of the 3D lung model [32]. As expected, epithelial cells formed a monolayer, MDMs were localized on the top of the epithelial monolayer and MDDCs at the basal surface of the insert. Further, the 3D lung model was exposed to fluorescently labeled NLCs, either with or without mannose, under submerged and pseudo-ALI conditions $\left(278 \mu \mathrm{g} / \mathrm{cm}^{2}\right)$ for $24 \mathrm{~h}$. With the latter, more realistic exposure conditions are mimicked compared with submerged exposures as alveolar epithelial cells produce surfactants that are released at the apical side of the inserts when cultured at the ALI conditions [26].

To confirm their biocompatibility, the cytotoxic effect of NPs exposure to the 3D lung model was assessed. Results revealed that NLCs did not alter the cell membrane integrity of the cells, neither under submerged nor pseudo-ALI conditions, which was in concordance with the expectedly high biocompatibility of lipid NPs [22]. Further, the pro-inflammatory response of the cells after NLCs exposure was also evaluated. As expected, the levels of IL-8 secretion were higher than the ones obtained for IL-1 $\beta$, and TNF- $\alpha$ because A549 cells produce a basal level of IL-8 in culture [42], but remained unaltered upon exposure to all the tested NLCs. This result was in agreement with previous studies using this model (either with alveolar or bronchial cells) to evaluate the potential cytotoxic and pro-inflammatory response upon exposure to biomedical NPs such as gold or hybrid lipid/polymer NPs [27,43].

To further check if mannosylated NLCs were more uptaken by MDMs than non-mannosylated NLCs, particle tracking and counting based on image processing was used. As the mannosylation process was used to actively target MDMs, a higher amount of C-M-NLCs agglomerates were expected. In fact, previous results demonstrated that M-NLCs are more efficiently internalized by AMs derived from bronchio-alveolar lavage of rats [44], and by mouse bone marrow-derived macrophages [22], than non-mannosylated NLCs. These studies were all performed using in vitro macrophages monocultures and therefore, reactions in more complex multicellular systems, such as the $3 \mathrm{D}$ tissue model employed herein, do not necessarily reflect cellular responses observed in 2D monocultures. Indeed, there is much lower number of MDMs in the 3D tissue model, in other words, approximately 1 per 40 epithelial cells $\left(1.19 \mathrm{MDMs} \times 10^{4}\right.$ cells $\left./ \mathrm{cm}^{2}\right)$ compared with $2.6 \mathrm{MDMs} \times 10^{5}$ cells $/ \mathrm{cm}^{2}$ used in previous monoculture suspension experiments (unpublished observation). Moreover, Guo et al. have demonstrated that mannosylation augments the cellular uptake of lipid NPs on A549 cell lines since the rapid proliferation of tumor cells increases their need for nutrients compared with normal cells, which results in the over-expression of lectin-like receptors that encompass high affinity for polysaccharide moieties including mannose [45]. Accordingly, it might be that non-mannosylated NLCs are less internalized by A549 and hence there are more NPs available to be taken up by MDMs. Thus, no significant differences between NLCs and M-NLCs agglomerates were observed.

Overall, this work contributed to the development of biocompatible nano-based systems for the delivery of drugs to TB-infected areas. Future work will include the encapsulation of anti-TB drugs with the challenges of reducing the required dose and minimize their dose-dependent side effects, which may contribute to decrease TB treatment duration and improve patient's compliance to therapy.

\section{Conclusion}

The application of nano-based systems for pulmonary drug delivery has been extensively explored to improve the treatment of respiratory infectious diseases, such as TB. Lipid NPs, namely NLCs, are promising drug delivery systems due to their biocompatibility, high drug loading capacity and stability. Additionally, NLCs can be produced in a controlled manner with appropriate size and morphology for lung deposition, and their surface can be decorated with mannose to specifically target AMs, the main reservoirs of bacteria involved in TB pathology.

Our work includes the development of non-mannosylated and mannosylated NLCs for the pulmonary delivery of anti-TB drugs, and the assessment of their cellular uptake in a 3D model of the alveolar epithelial tissue barrier. We have proven that exposure to all the tested NLCs formulations did not alter the cell membrane integrity nor the cellular morphology. In addition, the tested NLCs did not elicit cytotoxic and pro-inflammatory responses in the tissue in $24 \mathrm{~h}$ post exposure. Higher internalization in MDMs was expected for M-NLCs. However, we did not observe significant differences in the internalization of non- and mannosylated NLCs, both in submerged and pseudo-ALI conditions. This could be attributed to the fact that A549 over-express lectin-like receptors that have high affinity for mannose receptors, increasing receptor-mediated endocytosis of M-NLCs. Since non-mannosylated 
NLCs are less internalized by A549, there are more particles available to be taken up through nonspecific endocytosis by MDMs and thus, no significant differences between NLCs and M-NLCs were observed.

In sum, the in vitro biocompatible properties of lipid NPs were confirmed and thus, the developed NLCs can be considered for further testing as promising candidates for pulmonary drug delivery.

Summary points

- Nanostructured lipid carriers (NLC) were designed to facilitate drug delivery to tuberculosis (TB) infected areas.

- NLCs and mannosylated-NLCs (M-NLC) are uniform formulations and have an optimal size considering lung deposition (170-202 $\mathrm{nm}$ ).

- A 3D human lung model mimicking the alveolar epithelial tissue barrier was used to study the biocompatibility and cellular uptake of lipid nanoparticles (NP).

- Cell membrane integrity was maintained and no evidence of pro-inflammatory responses or morphological changes were observed $24 \mathrm{~h}$ post NP exposure.

- NLC and M-NLC were mostly distributed in the apical side of the lung tissue, both in monocyte-derived macrophages (MDM) and in epithelial cells.

- Higher internalization of M-NLCs was expected but no significant differences were observed between MDMs uptake of M-NLCs and NLCs.

- A549 overexpress lectin-like receptors that have high affinity for mannose receptors, increasing receptor-mediated endocytosis of M-NLCs. Since NLCs are less internalized by A549, there are more particles available to be uptaken through nonspecific endocytosis and thus, no significant differences between NLCs and M-NLCs uptake by MDMs were observed.

- In sum, the in vitro biocompatible properties of lipid NPs were confirmed and NLCs can be considered for further testing as candidates for pulmonary drug delivery.

\section{Acknowledgments}

The Adolphe Merkle Foundation is acknowledged, as well as the technical support by L Haeni, and H Barosova.

Financial \& competing interests disclosure

This work received financial support from the European Union (FEDER funds through COMPETE POCI-01-0145-FEDER-30624) and National Funds (FCT, Fundação para a Ciência e Tecnologia) through project PTDC/BTM-MAT/30624/2017. Joana Magalhães thanks FCT and POPH (Programa Operacional Potencial Humano) for the PhD grant SFRH/BD/110683/2015. This work was also supported by FCT through the FCT PhD Programs, specifically by the BiotechHealth Program (Doctoral Program on Cellular and Molecular Biotechnology Applied to Health Sciences). Marina Pinheiro thanks FCT for funding through program DL 57/2016 Norma transitória. Barbara Drasler was supported by the Peter und Traudl Engelhorn foundation. The authors have no other relevant affiliations or financial involvement with any organization or entity with a financial interest in or financial conflict with the subject matter or materials discussed in the manuscript apart from those disclosed.

No writing assistance was utilized in the production of this manuscript.

\section{Open access}

This work is licensed under the Attribution-NonCommercial-NoDerivatives 4.0 Unported License. To view a copy of this license, visit http://creativecommons.org/licenses/by-nc-nd/4.0/

\section{References}

Papers of special note have been highlighted as: $\bullet$ of interest; $\bullet \bullet$ of considerable interest

1. WHO. Global tuberculosis report 2018 (2018).

2. Zumla A, Chakaya J, Centis R et al. Tuberculosis treatment and management-an update on treatment regimens, trials, new drugs, and adjunct therapies. Lancet Respir. Med. 3(3), 220-234 (2015).

3. Zazo H, Colino CI, Lanao JM. Current applications of nanoparticles in infectious diseases. J. Control. Release 224, 86-102 (2016).

4. Aristoff PA, Garcia GA, Kirchhoff PD, Showalter HD. Rifamycins-obstacles and opportunities. Tuberculosis. 90(2), 94-118 (2010).

5. Zumla A, Nahid P, Cole ST. Advances in the development of new tuberculosis drugs and treatment regimens. Nat. Rev. Drug Discov. 12(5), 388-404 (2013).

6. Magalhães J, Vieira A, Pinto $S$ et al. New approaches from nanomedicine and pulmonary drug delivery for the treatment of tuberculosis. In: Nanoparticles in the Life Sciences and Biomedicine. Neves AR, Resi S (Eds). Pan Stanford Publishing, NY, USA, 197-234 (2018). 
7. Rothen-Rutishauser B, Blank F, Muhlfeld C, Gehr P. In vitro models of the human epithelial airway barrier to study the toxic potential of particulate matter. Expert Opin. Drug Metab. Toxicol. 4(8), 1075-1089 (2008).

8. Hidalgo A, Cruz A, Perez-Gil J. Pulmonary surfactant and nanocarriers: toxicity versus combined nanomedical applications. Biochim. Biophys. Acta Biomembr. 1859(9 Pt B), 1740-1748 (2017).

9. Oberdorster G. Lung dosimetry: pulmonary clearance of inhaled particles. Aerosol Sci. Technol. 18(3), 279-289 (2007).

- Provides a brief review of the deposition, clearance and retention mechanisms of inhalable compounds throughout the respiractory tract.

10. Geiser M, Kreyling WG. Deposition and biokinetics of inhaled nanoparticles. Part Fibre Toxicol. 7(1), 2 (2010).

11. Oberdorster G, Oberdorster E, Oberdorster J. Nanotoxicology: an emerging discipline evolving from studies of ultrafine particles. Environ. Health Perspect. 113(7), 823-839 (2005).

12. Patton JS, Byron PR. Inhaling medicines: delivering drugs to the body through the lungs. Nat. Rev. Drug Discov. 6(1), 67-74 (2007).

13. Gehr P, Bachofen M, Weibel ER. The normal human lung: ultrastructure and morphometric estimation of diffusion capacity. Respir. Physiol. 32(2), 121-140 (1978).

14. Lieber M, Smith B, Szakal A, Nelson-Rees W, Todaro G. A continuous tumor-cell line from a human lung carcinoma with properties of type II alveolar epithelial cells. Int. J. Cancer 17(1), 62-70 (1976).

15. Kopf M, Schneider C, Nobs SP. The development and function of lung-resident macrophages and dendritic cells. Nat. Immunol. 16(1), 36-44 (2015).

16. Mohammadian M, Pourmehran O. CFPD simulation of magnetic drug delivery to a human lung using an SAW nebulizer. Biomech. Model Mechanobiol. 18(3), 547-562 (2019).

17. Mehanna MM, Mohyeldin SM, Elgindy NA. Respirable nanocarriers as a promising strategy for antitubercular drug delivery. J. Control. Release 187, 183-197 (2014).

18. Patil TS, Deshpande AS. Nanostructured lipid carriers-based drug delivery for treating various lung diseases: a state-of-the-art review. Int. J. Pharm. 547(1-2), 209-225 (2018).

-. Recent review that demonstrated the application of nanostructured lipid carriers (NLC) for pulmonary drug delivery.

19. Abdelaziz HM, Gaber M, Abd-Elwakil MM et al. Inhalable particulate drug delivery systems for lung cancer therapy: nanoparticles, microparticles, nanocomposites and nanoaggregates. J. Control. Release 269, 374-392 (2018).

20. Jain A, Agarwal A, Majumder S et al. Mannosylated solid lipid nanoparticles as vectors for site-specific delivery of an anti-cancer drug. $J$. Control. Release 148(3), 359-367 (2010).

- Protocol for mannose coating of lipid NPs.

21. Pinheiro M, Ribeiro R, Vieira A, Andrade F, Reis S. Design of a nanostructured lipid carrier intended to improve the treatment of tuberculosis. Drug Des. Devel. Ther. 10, 2467-2475 (2016).

22. Vieira AC, Magalhaes J, Rocha S et al. Targeted macrophages delivery of rifampicin-loaded lipid nanoparticles to improve tuberculosis treatment. Nanomedicine. 12(24), 2721-2736 (2017).

-• Proof of concept that macrophage-targeted NLCs are efficiently internalized by BMDMs in vitro.

23. Srivastava S, Ernst JD, Desvignes L. Beyond macrophages: the diversity of mononuclear cells in tuberculosis. Immunol. Rev. 262(1), 179-192 (2014).

24. Goenka A, Casulli J, Hussell T. Mycobacterium tuberculosis joyrides alveolar macrophages into the pulmonary interstitium. Cell Host Microbe 24(3), 331-333 (2018).

25. Rothen-Rutishauser BM, Kiama SG, Gehr P. A three-dimensional cellular model of the human respiratory tract to study the interaction with particles. Am. J. Respir. Cell Mol. Biol. 32(4), 281-289 (2005).

-• Description and characterization of the triple cell co-culture model designed to simulate the human alveolar epithelial barrier.

26. Blank F, Rothen-Rutishauser BM, Schurch S, Gehr P. An optimized in vitro model of the respiratory tract wall to study particle cell interactions. J. Aerosol Med. 19(3), 392-405 (2006).

-. Proof of concept that the triple cell co-culture model is appropriate to study particles-cell interactions.

27. Fytianos K, Chortarea S, Rodriguez-Lorenzo L et al. Aerosol delivery of functionalized gold nanoparticles target and activate dendritic cells in a 3d lung cellular model. ACS Nano 11(1), 375-383 (2017).

28. Iemsam-Arng J, Ketchart O, Rattana-Amron T, Wutikhun T, Tapaneeyakorn S. Modified NLC-loaded coumarin for pharmaceutical applications: the improvement of physical stability and controlled release profile. Pharm. Dev. Technol. 21(8), 1015-1022 (2016).

29. Sallusto F, Cella M, Danieli C, Lanzavecchia A. Dendritic cells use macropinocytosis and the mannose receptor to concentrate macromolecules in the major histocompatibility complex class II compartment: downregulation by cytokines and bacterial products. J. Exp. Med. 182(2), 389-400 (1995).

30. Drasler B, Kucki M, Delhaes F et al. Single exposure to aerosolized graphene oxide and graphene nanoplatelets did not initiate an acute biological response in a 3D human lung model. Carbon 137, 125-135 (2018). 
31. Kabakov AE, Gabai VL. Cell death and survival assays. Methods Mol. Biol. 1709, 107-127 (2018).

32. Septiadi D, Bourquin J, Durantie E, Petri-Fink A, Rothen-Rutishauser B. A novel sample holder for 4D live cell imaging to study cellular dynamics in complex 3D tissue cultures. Sci. Rep. 8(1), 9861 (2018).

33. Tinevez JY, Perry N, Schindelin J et al. TrackMate: An open and extensible platform for single-particle tracking. Methods 115, 80-90 (2016).

34. Neves AR, Lucio M, Martins S, Lima JL, Reis S. Novel resveratrol nanodelivery systems based on lipid nanoparticles to enhance its oral bioavailability. Int. J. Nanomedicine 8, 177-187 (2013).

35. Ferreira M, Chaves LL, Lima SA, Reis S. Optimization of nanostructured lipid carriers loaded with methotrexate: a tool for inflammatory and cancer therapy. Int. J. Pharm. 492(1-2), 65-72 (2015).

36. Granja A, Vieira AC, Chaves LL et al. Folate-targeted nanostructured lipid carriers for enhanced oral delivery of epigallocatechin-3-gallate. Food Chem. 237, 803-810 (2017).

37. Vieira AC, Chaves LL, Pinheiro M, Ferreira D, Sarmento B, Reis S. Design and statistical modeling of mannose-decorated dapsone-containing nanoparticles as a strategy of targeting intestinal M-cells. Int. J. Nanomedicine 11, 2601-2617 (2016).

38. Heyder J, Gebhart J, Rudolf G, Schiller CF, Stahlhofen W. Deposition of particles in the human respiratory tract in the size range

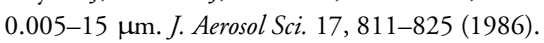

39. Costa A, Pinheiro M, Magalhães J et al. The formulation of nanomedicines for treating tuberculosis. Adv. Drug Deliv. Rev. 102, 102-115 (2016).

40. Danaei M, Dehghankhold M, Ataei $\mathrm{S}$ et al. Impact of particle size and polydispersity index on the clinical applications of lipidic nanocarrier systems. Pharmaceutics 10(2), pii: E57 (2018).

41. Muller RH, Jacobs C, Kayser O. Nanosuspensions as particulate drug formulations in therapy. rationale for development and what we can expect for the future. Adv. Drug Deliv. Rev. 47(1), 3-19 (2001).

42. Standiford TJ, Kunkel SL, Basha MA et al. Interleukin- 8 gene expression by a pulmonary epithelial cell line. A model for cytokine networks in the lung. J. Clin. Invest. 86(6), 1945-1953 (1990).

43. D'angelo I, Costabile G, Durantie E et al. Hybrid lipid/polymer nanoparticles for pulmonary delivery of siRNA: development and fate upon in vitro deposition on the human epithelial airway barrier. J. Aerosol Med. Pulm. Drug Deliv. 31(3), 170-181 (2018).

44. Song X, Lin Q, Guo L et al. Rifampicin loaded mannosylated cationic nanostructured lipid carriers for alveolar macrophage-specific delivery. Pharm. Res. 32(5), 1741-1751 (2015).

45. Guo Y, Liu X, Sun X, Zhang Q, Gong T, Zhang Z. Mannosylated lipid nano-emulsions loaded with lycorine-oleic acid ionic complex for tumor cell-specific delivery. Theranostics 2(11), 1104-1114 (2012). 
